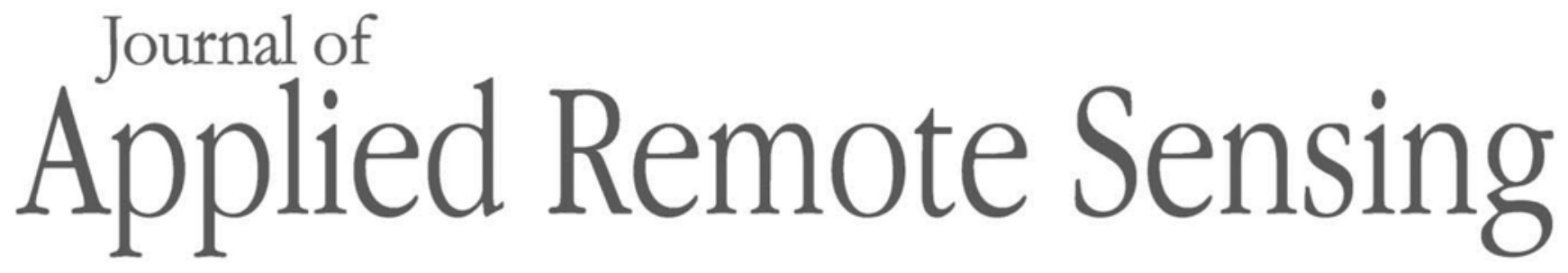

RemoteSensing.SPIEDigitalLibrary.org

\title{
Assessing the impact of porosity variations on the reflectance and transmittance of natural sands
}

\author{
Gladimir V. G. Baranoski \\ Bradley W. Kimmel \\ Petri Varsa
}




\title{
Assessing the impact of porosity variations on the reflectance and transmittance of natural sands
}

\author{
Gladimir V. G. Baranoski,* Bradley W. Kimmel, and Petri Varsa \\ University of Waterloo, David R. Cheriton School of Computer Science, \\ Natural Phenomena Simulation Group, Waterloo, Ontario, Canada
}

\begin{abstract}
Porosity is a fundamental characteristic of naturally occurring sand-textured soils, commonly referred to as natural sands, found in a wide range of landscapes, from beaches to dune fields. As a primary determinant of the density and permeability of sediments, it represents a pivotal element in geophysical studies involving basin modeling and the optical dating of sand deposits formed in areas subjected to erosion like coasts and deltas. It is also of interest for geoaccoustics and geochemical research on sediment transport and water diffusion properties of these deposits, as well as for agricultural and ecological investigations on the germination of light-sensitive seeds and the photochemical transformation of substances (e.g., pesticides) that may be present in these soils. Despite the importance of these applications, however, the remote estimation of porosity and the quantification of its effects on the light penetration profiles of natural sands remain elusive tasks. In this work, we tackle one of the major obstacles in this interdisciplinary area of research, namely the relative scarcity of experimental information due to technical constraints associated with traditional laboratory procedures. More specifically, we systematically examine the impact of porosity variations on the reflectance and transmittance of natural sands (in the 400 to $1000 \mathrm{~nm}$ region of the light spectrum) through controlled in silico experiments supported by measured data. Our findings are expected to strengthen the knowledge basis required for advances in this area and contribute to the development of technologies aimed at the effective monitoring and prediction of environmentally triggered changes affecting these soils. (C) The Authors. Published by SPIE under a Creative Commons Attribution 4.0 Unported License. Distribution or reproduction of this work in whole or in part requires full attribution of the original publication, including its DOI. [DOI: 10.1117/1.JRS.13.024522]
\end{abstract}

Keywords: sand; porosity; water saturation; sphericity; roundness; reflectance; transmittance; predictive simulations.

Paper 180992 received Dec. 21, 2018; accepted for publication May 9, 2019; published online Jun. 10, 2019.

\section{Introduction}

Soils are mostly composed of mineral particles (grains) of weathered rock immersed in a medium (e.g., air and/or water). ${ }^{1}$ They are classified according to the size distribution of their constituent particles. ${ }^{2}$ This is accomplished initially by assigning individual particles into classes, called soil separates, according to their size. The relative masses of each soil separate are then compared to determine the texture of a soil sample. ${ }^{3}$

Various agencies have differing definitions for soil separates and textures. In this work, we employ the system developed by the US Department of Agriculture. ${ }^{4}$ It defines three soil separates called sand (containing the largest particles), silt, and clay (containing the smallest particles). Naturally occurring sand-textured soils, henceforth referred to as natural sands or sand deposits, contain at least $85 \%$ of sand-sized particles. ${ }^{4}$ These soils comprise over $20 \%$ of the Earth's land surface. ${ }^{5}$

The porosity of a soil sample corresponds to the fraction of its total volume occupied by its pore space (the volume not occupied by its constituent particles). ${ }^{6}$ The porosity of natural sands typically varies between 0.35 and $0.5,2,7$ with values as low as $0.196^{7,8}$ and as high as $0.66^{7,9}$ being also reported in the literature. Incidentally, porosity values can also appear in related works expressed in terms of percentages.

*Address all correspondence to Gladimir V. G. Baranoski, E-mail: gvgbaran@curumin.math.uwaterloo.ca 
Porosity is a primary determinant of the density and permeability of sediments. It represents an essential input for basin modeling and geophysical studies involving erosion of regions more vulnerable to environmental changes, such as coastal areas and river deltas. ${ }^{7}$ For example, the effective optical dating ${ }^{10}$ of sand deposits found in these regions ${ }^{11}$ relies on the careful appraisal of their light penetration (transmission) profiles. These are affected by the packing conditions of these deposits, ${ }^{12}$ which are directly associated with their porosity. The impact of porosity on light penetration is also relevant for agricultural and ecological investigations on the germination of light-sensitive seeds ${ }^{13-15}$ and the photochemical transformation of substances (e.g., pesticides) that may be present in these soils. ${ }^{16}$

It is also worth noting that the porosity of a sand deposit can be used to calculate its wet bulk density. ${ }^{17}$ This quantity, in turn, is of interest for a variety of geoacoustics ${ }^{18}$ and geochemical ${ }^{17}$ investigations aiming at the understanding of sediment transport and water diffusion properties of natural sands. This connection between porosity and water flow is also one of the focal points of hydrological studies involving the permeability of aquifers. ${ }^{17}$

These applications underscore the importance of obtaining reliable porosity estimations for target sand deposits. This often involves measuring the porosity of samples collected from these deposits. The physical handling of the samples during these procedures can result in grain breakage as well as pore space disturbance associated with grain-to-grain rearrangements and water loss (in the case of wet samples). ${ }^{8,17,18}$ These alterations in the samples' interstitial structure can, in turn, introduce experimental biases that undermine the accuracy of porosity measurements. More recently, in situ measurement approaches, such as the nuclear densimeter method, ${ }^{7}$ are being proposed to eliminate the physical handling of samples.

When it comes to the remote estimation of porosity and the quantification of its effects on the light penetration profiles of natural sands, however, there is still a long way to go. This may be largely attributed to the relative scarcity of experimental investigations on the putative dependence of these soils' spectral responses on their porosity. ${ }^{19,20}$ In order to achieve tangible advances in this area, we believe that it is necessary to examine this dependence more closely.

This aspect has prompted the research described in this paper, which is an updated and extended version of a conference presentation. ${ }^{21}$ Here, we investigate the impact of porosity variations on the reflectance and transmittance of natural sands in the spectral region of interest from 400 to $1000 \mathrm{~nm}$. Our primary goals are to assess the putative dependence of the spectral signatures of these soils on their porosity and to examine the effects of porosity variations on their light penetration profiles.

To overcome the difficulties inherent to conventional experimental practices, we employ an in silico experimental approach supported by measured data and centered on predictive computer simulations. These are performed using a first-principles hyperspectral model for particulate materials, known as spectral light transport model for sand (SPLITS), ${ }^{22}$ that takes into account the specific mineralogical and morphological characteristics of natural sands.

This approach was also chosen for enabling us to perform controlled experiments involving light reflection and transmission by natural sand samples. In other words, we can assign different values to selected material characterization parameters and analyze their effects on the samples' reflectance and transmittance while keeping all other parameters fixed. We remark that such controlled experiments are cumbersome to be effectively carried out using traditional laboratory set-ups.

Our findings highlight radiometric trends that need to be taken into account in the development of technologies aimed at the correct interpretation of variations in the spectral signatures of natural sands and at the reliable estimation of their porosity and light penetration profiles. These technologies, in turn, will enable researchers to make inroads into the applications mentioned earlier, notably those associated with the effective monitoring and prediction of environmentally triggered changes in natural sand deposits.

The remainder of this paper is organized as follows. In Sec. 2, we describe the in silico experimental framework employed in our investigation. In Sec. 3, we present and discuss our results. Finally, in Sec. 4, we conclude the paper and outline directions for future research. 


\section{In Silico Experimental Framework}

Our investigation focuses on surficial, noncemented sand deposits. Besides variations on porosity $(P)$, it takes into account variations on other sand characteristics, namely the degree of water saturation $(S)$ and grain shape. ${ }^{22}$ The former represents the fraction of pore space occupied by water. The latter is usually specified by two parameters: roundness $(R)$ and sphericity $(\Psi){ }^{23}$ While roundness indicates the measure of detail in the features on the grain surface, sphericity refers to the extent to which the grain approaches a spherical shape. ${ }^{24}$

The reflection, transmission, and absorption profiles of natural sands are largely determined by light interactions with attenuation agents (scatterers and absorbers), notably iron oxides (e.g., hematite, goethite, and magnetite), present in these soils. These interactions are fully taken into account by the SPLITS model, ${ }^{22}$ which obeys the principle of energy conservation, ${ }^{25}$ i.e., reflectance, transmittance, and absorptance values obtained at a given wavelength sum to unity. Thus, it enables the predictive computation of spectral reflectance, transmittance, and absorptance curves for these particulate materials. Since the focus of this work is on changes in the light reflection and transmission profiles of natural sands in response to variations in their porosity, our in silico experiments are centered on the computation of the corresponding reflectance and transmittance curves for selected samples of these soils.

Besides the amounts of iron oxides present in natural sands, SPLITS also accounts for their different distribution patterns within these soils. ${ }^{22}$ These light attenuation agents may occur as pure particles, ${ }^{26}$ as contaminants mixed with the parent material, ${ }^{27}$ or as coatings (within a mineral matrix) formed on the grains during wind transport. ${ }^{28}$ Normally, the coating matrix is composed of kaolinite and/or illite, ${ }^{29}$ with the former (employed in this investigation) being more prevalent in natural sands. ${ }^{30,31}$ Also, in these soils, the parent (grain core) material is typically a mineral like quartz or calcite, with quartz (employed in this investigation) being the most common. $^{32}$

As baseline modeled data for our investigation, we computed directional-hemispherical reflectance curves for two selected natural sand samples with distinct morphological and mineralogical characteristics. These curves were obtained using actual reflectance measurements for these samples ${ }^{33}$ as references. These measurements, which were made available in the US Army Topographic Engineering Center (TEC) database, ${ }^{33}$ correspond to a sample from a red (hematiterich) dune in Australia (TEC \#10019201) and a sample from a magnetite-rich beach site in Peru (TEC \#10039240). Based on the descriptions of these samples,${ }^{33}$ we assumed that the presence of clay-sized particles and water was negligible.

For the computation of the baseline reflectance curves, besides considering a dry state $(S=0)$, we employed mean values for the porosity $(P=0.425)$, grain roundness $(R=0.482)$, and grain sphericity $(\Psi=0.798)$ found in the literature. ${ }^{2,23}$ The remaining model parameter values employed to characterize the selected natural sand samples are given in Table 1. Note that the percentages of the sand-sized and silt-sized particles depicted in Table 1 are used to compute their dimensions during the simulations ${ }^{24}$ using a particle size distribution provided by Shirazi et al. ${ }^{3}$ The corresponding particle dimensions are provided in Table 2.

Table 1 Parameter values used to characterize the natural sand samples considered in this investigation. The texture of the samples is described by the percentages of sand $\left(s_{a}\right)$ and silt $\left(s_{i}\right)$. The particle type distributions employed in the simulations are given in terms of the percentages of pure $\left(\mu_{p}\right)$, mixed $\left(\mu_{m}\right)$, and coated $\left(\mu_{c}\right)$ grains. The parameter $r_{h g}$ corresponds to the ratio between the mass fraction of hematite to $\vartheta_{h g}$ (the total mass fraction of hematite and goethite). The parameter $\vartheta_{m}$ represents the mass fraction of magnetite, which is assumed to appear as pure particles. $^{24}$

\begin{tabular}{lcccccccc}
\hline \hline & $s_{a}$ & $s_{i}$ & $\mu_{p}$ & $\mu_{m}$ & $\mu_{c}$ & $r_{h g}$ & $\vartheta_{h g}$ & $\vartheta_{m}$ \\
\hline Australian dune & 85 & 15 & 0 & 90 & 10 & 0.75 & 0.01 & 0.0 \\
Peruvian beach & 92.8 & 7.2 & 50 & 0 & 50 & 0.35 & 0.05 & 0.17 \\
\hline \hline
\end{tabular}


Table 2 Average dimensions (given in $\mathrm{mm}$ ) of the major axes $m_{a}$ and $m_{i}$ that respectively define the ellipsoids used to represent the sand-sized and the silt-sized particles forming the natural sand samples considered in this investigation.

\begin{tabular}{lcc}
\hline \hline & $m_{a}$ & $m_{i}$ \\
\hline Australian dune & 0.236 & 0.045 \\
Peruvian beach & 0.265 & 0.044 \\
\hline \hline
\end{tabular}

The SPLITS model employs a geometrical-optics formulation in which the light interactions with a given sand sample are simulated using rays. These, in turn, can be associated with any wavelength $(\lambda)$. Hence, SPLITS can provide reflectance and transmittance curves with different spectral resolutions. For consistency, all modeled curves depicted in this work have a spectral resolution of $5 \mathrm{~nm}$. These curves were obtained using a virtual spectrophotometer. ${ }^{34}$ In their computation, we considered $10^{6}$ sample rays (per sampled wavelength) to obtain asymptotically convergent readings,${ }^{34}$ and an angle of incidence of 0 deg for consistency with the actual measurement set-up employed by Rinker et al. ${ }^{22,33}$ to obtain the reference reflectance curves.

The reflectance and transmittance of sand samples have opposite behaviors with respect to changes in their thickness. While reflectance rapidly reaches a plateau (occasionally referred to as "infinite reflectance"16), when one considers increasing thickness values, transmittance rapidly tends to zero. ${ }^{16}$ In the case of natural sand samples characterized by the presence of strong light absorbers (e.g., iron oxides), this plateau is reached with thickness values on the order of millimeters. ${ }^{35}$ Accordingly, the reflectance of natural sand deposits is guaranteed to arrive at this plateau since these deposits normally have fairly large depths on the order of meters. Thus, without loss of generality, we consider a sample thickness of $1 \mathrm{~m}$ in the computation of the modeled reflectance curves depicted in this investigation.

For the computation of the transmittance curves, we considered a sample thickness of $1 \mathrm{~mm}$. This value was selected for two reasons. First, it corresponds to a standard light penetration depth relevant for agricultural and ecological applications. ${ }^{13-15,36}$ Second, it enables the detection of relatively large transmittance signals, which facilitates their analysis.

To streamline the reproduction of our in silico experimental results, we made SPLITS available online ${ }^{37}$ via a model distribution system. ${ }^{38}$ Through this system, researchers can specify experimental conditions (e.g., angle of incidence and spectral range) and values for sand characterization parameters using a web interface, ${ }^{37}$ and receive customized simulation results. In addition, we also made the supporting data (e.g., refractive index and extinction coefficient curves) used in our investigation available online. ${ }^{39}$

As it can be observed in the graphs presented in Fig. 1, the baseline modeled reflectance curves computed for the two selected natural sand samples show a close agreement with their measured counterparts. Accordingly, the reflectance and transmittance curves resulting from variations in porosity, grain roundness, and grain sphericity were also obtained using the characterization datasets provided in Table 1 . However, for the computation of these curves, we employed lower and upper bounds for $P(0.196$ and 0.66$),{ }^{7} S(0$ and 1$), R(0.2$ and 0.7$),{ }^{23}$ and $\Psi(0.6$ and 0.95$),{ }^{23}$ respectively. Note that $S$ equal to 1 represents a pore space completely filled with water, $R$ equal to 0.7 corresponds to the smoothest grains, and $\Psi$ equal to 0.95 describes grains whose geometry is the closest to that of a sphere.

Finally, in order to quantify the changes in light reflection resulting from porosity variations, we compute the mean relative differences (MRDs) between the corresponding reflectance curves with respect to the visible (400 to $700 \mathrm{~nm}$ ) and near-infrared (700 to $1000 \mathrm{~nm}$ ) portions of the spectral region of interest. This metric, which is expressed in terms of percentage, is calculated using the following formula:

$$
\operatorname{MRD}_{\rho}=\frac{1}{N} \sum_{i=1}^{N} \frac{\left|\rho_{P=0.196}\left(\lambda_{i}\right)-\rho_{P=0.66}\left(\lambda_{i}\right)\right|}{\rho_{P=0.66}\left(\lambda_{i}\right)} \times 100,
$$


(a)

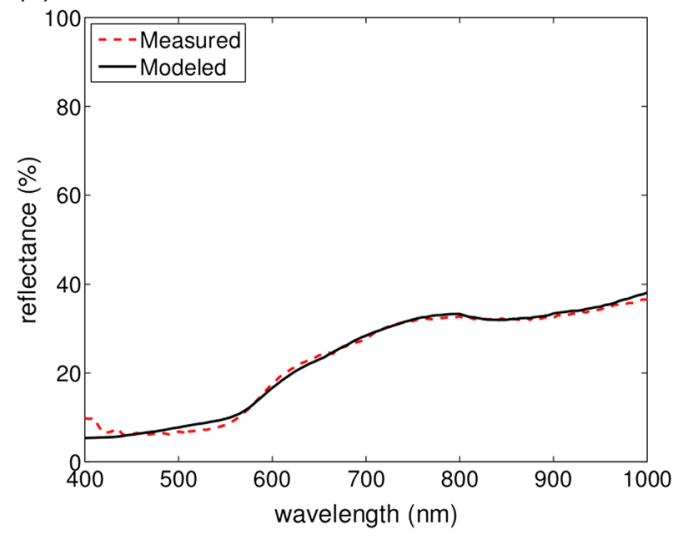

(b)

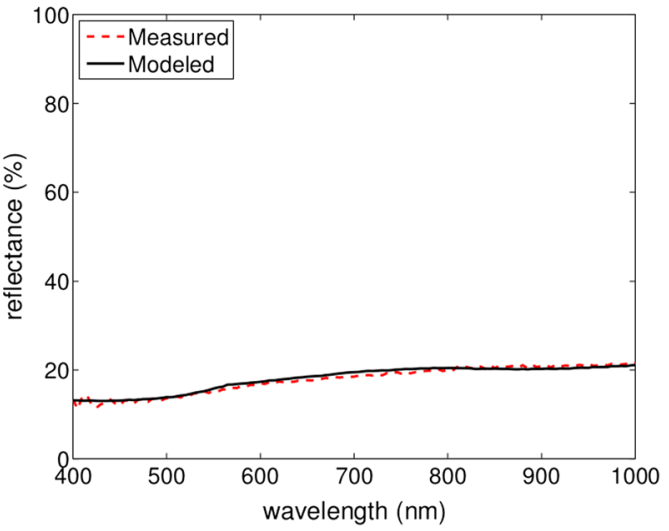

Fig. 1 Measured and modeled reflectance curves for the two natural sand samples employed as baseline references in this investigation. (a) A hematite-rich (red) dune in Australia (TEC \#10019201). (b) A magnetite-rich (dark) beach site in Peru (TEC \#10039240). The measured curves were obtained from the U.S. Army Topographic Engineering Center (TEC) database. ${ }^{33}$ The modeled curves were computed using the SPLITS model. ${ }^{22,37}$

where $\rho_{P=0.196}$ and $\rho_{P=0.66}$ correspond to the reflectance curves obtained considering porosity set to 0.196 and 0.66 , respectively, and $N$ is the total number of wavelengths sampled with a $5 \mathrm{~nm}$ resolution within the selected portion of the spectral region of interest.

Similarly, to quantify the changes in light transmission resulting from porosity variations, we computed the MRDs between the corresponding transmittance curves $\left(\tau_{P=0.196}\right.$ and $\left.\tau_{P=0.66}\right)$. This metric, which is also expressed in terms of percentage, is calculated using the formula below:

$$
\operatorname{MRD}_{\tau}=\frac{1}{N} \sum_{i=1}^{N} \frac{\left|\tau_{P=0.196}\left(\lambda_{i}\right)-\tau_{P=0.66}\left(\lambda_{i}\right)\right|}{\tau_{P=0.66}\left(\lambda_{i}\right)} \times 100 .
$$

\section{Results and Discussion}

Initially, we assessed the impact of porosity variations on the selected natural sand samples' reflectance. We then proceeded to assess the impact of these porosity variations on the samples' transmittance. These assessments were carried out through in silico light reflection and transmission experiments whose results are presented and discussed in Secs. 3.1 and 3.2, respectively. Practical issues associated with the fidelity of our findings are addressed in Sec. 3.3.

\subsection{Light Reflection Experiments}

In our first set of light reflection experiments, we examined how the samples' reflectance is affected by the combined effects of variations in their porosity and degree of water saturation. The resulting plots depicted in Fig. 2 show the expected reflectance decrease associated with the increase in the degree of water saturation. ${ }^{16,40,41}$ They also show relatively small changes associated with the different porosity values. More precisely, in the case of the Australian dune sample, which was modeled considering smaller particle dimensions, one can observe a minor reflectance decrease from 400 to $950 \mathrm{~nm}$ when $P$ is increased from 0.196 to 0.66 and $S$ is set to 0 [Fig. 2(a)]. In addition, one can also observe a minor increase in the reflectance between 950 and $1000 \mathrm{~nm}$ under the same conditions. When $S$ is set to 1 [Fig. 2(c)], the reflectance changes become slightly smaller in the visible portion of the spectral region of interest and slightly larger in the near-infrared portion. These observations are corroborated by the corresponding $\mathrm{MRD}_{\rho}$ values provided in Table 3. In the case of the Peruvian beach sample, which was modeled considering larger particle dimensions, one can observe a minor reflectance decrease along the entire spectral region of interest when $P$ is increased from 0.196 to 0.66 and 
(a)

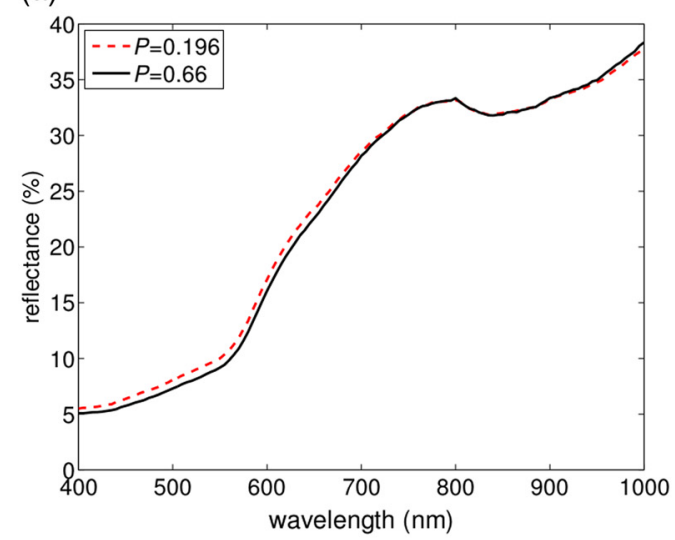

(c)

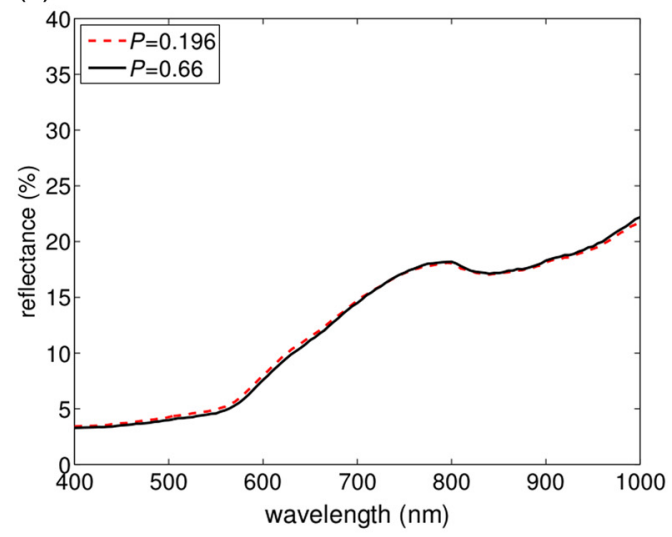

(b)

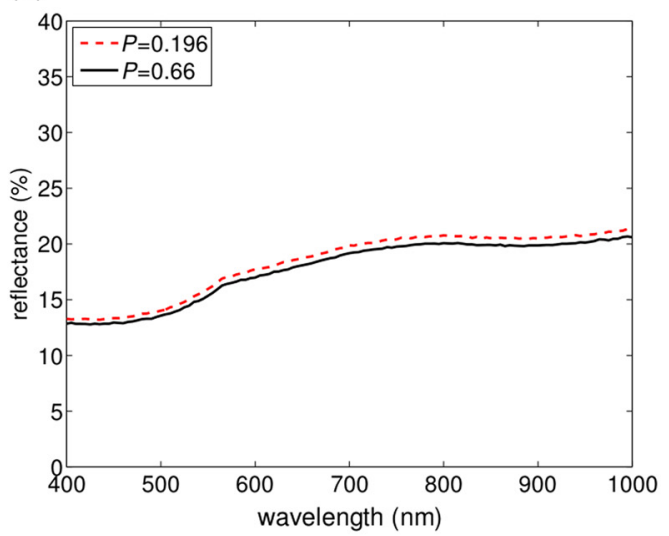

(d)

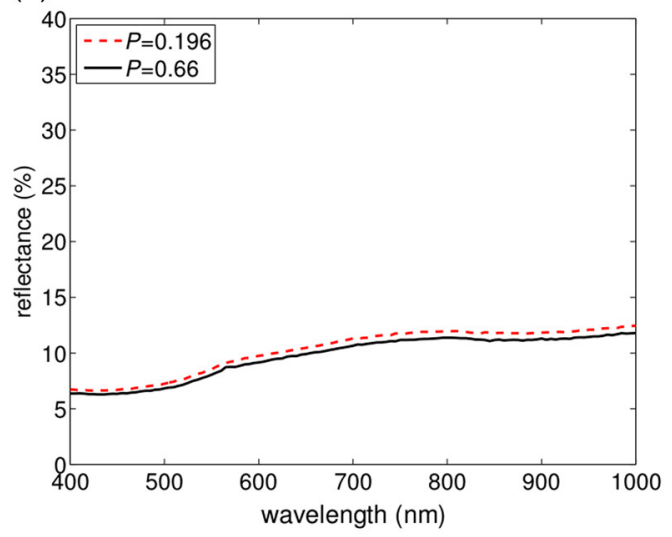

Fig. 2 Comparisons of modeled reflectance curves obtained for the Australian dune sample $[(\mathrm{a})$ and $(\mathrm{c})]$ and the Peruvian beach sample $[(\mathrm{b})$ and $(\mathrm{d})]$ considering variations in porosity $(P)$ and degree of water saturation (S). (a) and (b) $S=0$. (c) and (d) $S=1$.

Table $3 \mathrm{MRD}_{\rho}$ values (given in percentages) computed for the modeled reflectance curves depicted in Fig. 2, which were obtained considering variations in porosity $(P=0.196$ and $P=0.66)$ and degree of water saturation $(S=0$ and $S=1)$.

\begin{tabular}{lccccc}
\hline \multirow{2}{*}{$\begin{array}{l}\text { Characterization } \\
\text { Parameter }\end{array}$} & \multicolumn{2}{c}{ Australian dune } & & \multicolumn{2}{c}{ Peruvian beach } \\
\cline { 2 - 3 } \cline { 5 - 6 } & Visible & Near-infrared & & Visible & Near-infrared \\
\hline$S=0$ & 7.71 & 0.49 & & 3.57 & 3.30 \\
$S=1$ & 4.94 & 0.83 & & 5.92 & 5.39 \\
\hline \hline
\end{tabular}

$S$ is set to 0 [Fig. 2(c)]. When $S$ is set to 1 [Fig. 2(d)], the reflectance changes become slightly larger. These observations are also corroborated by the corresponding $\mathrm{MRD}_{\rho}$ values provided in Table 3.

In our second set of light reflection experiments, we examined how the samples' reflectance is affected by the combined effects of variations in their porosity and in the roundness of their constituent grains. The resulting plots depicted in Fig. 3 again show relatively small changes associated with the different porosity values. In the case of the Australian dune sample, these changes are more noticeable in the visible portion of the spectral region of interest. In the case of the Peruvian beach sample, however, these changes are noticeable along the entire spectral region of interest. These plots also show that, for both samples, the magnitude of these changes is slightly reduced when $R$ is set to 0.7 . This observation is corroborated by the corresponding $\mathrm{MRD}_{\rho}$ values provided in Table 4. 
(a)

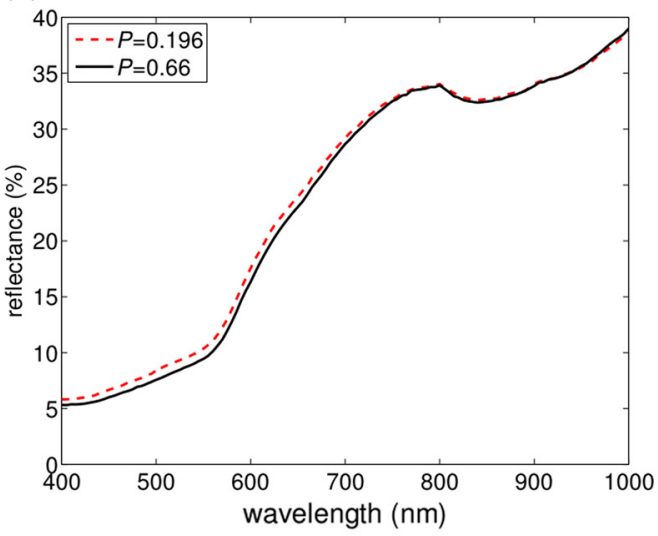

(c)

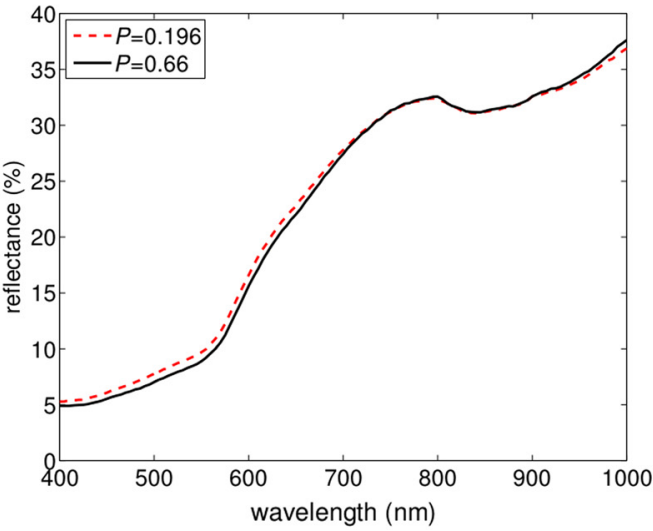

(b)

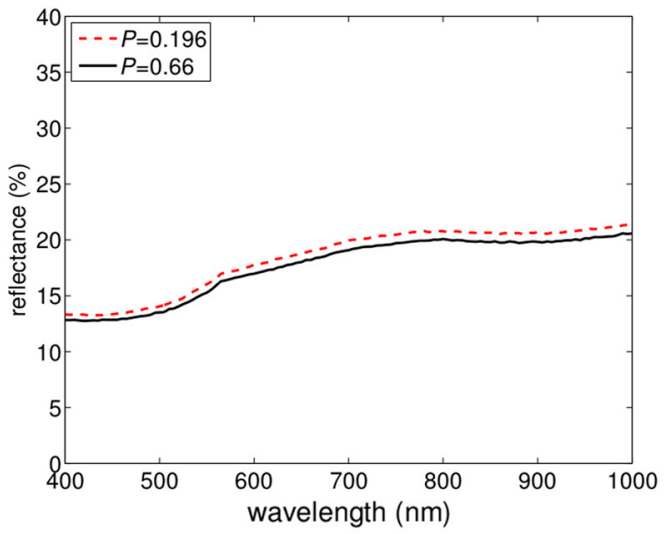

(d)

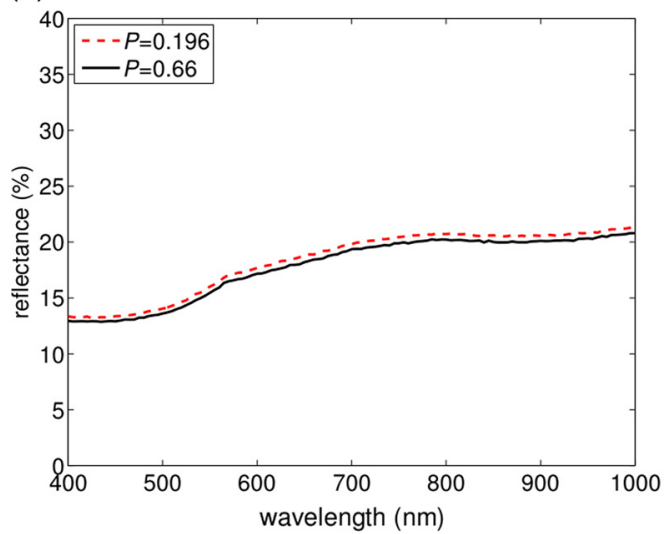

Fig. 3 Comparisons of modeled reflectance curves obtained for the Australian dune sample $[(\mathrm{a})$ and $(\mathrm{c})]$ and the Peruvian beach sample $[(\mathrm{b})$ and $(\mathrm{d})]$ considering variations in porosity $(P)$ and grain roundness $(R)$. (a) and (b) $R=0.2$. (c) and (d) $R=0.7$.

Table $4 \mathrm{MRD}_{\rho}$ values (given in percentages) computed for the modeled reflectance curves depicted in Fig. 3, which were obtained considering variations in porosity $(P=0.196$ and $P=0.66)$ and grain roundness $(R=0.2$ and $R=0.7)$.

\begin{tabular}{lccccc}
\hline \hline \multirow{2}{*}{$\begin{array}{l}\text { Characterization } \\
\text { Parameter }\end{array}$} & \multicolumn{2}{c}{ Australian dune } & & \multicolumn{2}{c}{ Peruvian beach } \\
\cline { 2 - 3 } \cline { 5 - 6 } & Visible & Near-infrared & & Visible & Near-infrared \\
\hline$R=0.2$ & 7.90 & 0.63 & & 4.15 & 3.95 \\
$R=0.7$ & 7.48 & 0.55 & & 3.12 & 2.65 \\
\hline \hline
\end{tabular}

In our third set of light reflection experiments, we examined how the samples' reflectance is affected by the combined effects of variations in their porosity and in the sphericity of their constituent grains. Similar to the previous sets of light reflection experiments, the resulting plots depicted in Fig. 4 show relatively small quantitative changes associated with the different porosity values. This set of experiments, however, led to the detection of markedly distinct qualitative trends. In the case of the Australian dune sample, while one can observe a minor reflectance decrease in the region from 400 to $\approx 620 \mathrm{~nm}$ when $P$ is increased from 0.196 to 0.66 and $\Psi$ is set to 0.6 [Fig. 4(a)], one can observe a noticeable reflectance increase in the region from $\approx 620$ to $1000 \mathrm{~nm}$. When $\Psi$ is set to 0.95 [Fig. 4(c)], on the other hand, one can observe a reflectance reduction along the entire spectral region of interest, albeit more pronounced in the visible portion. In the case of the Peruvian beach sample, one can observe a minor reflectance decrease along the entire spectral region of interest when $P$ is increased from 0.196 to 0.66 and 
(a)

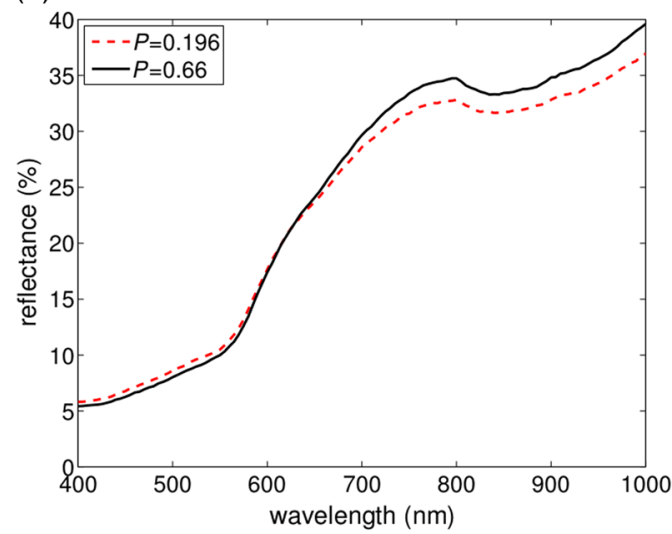

(c)

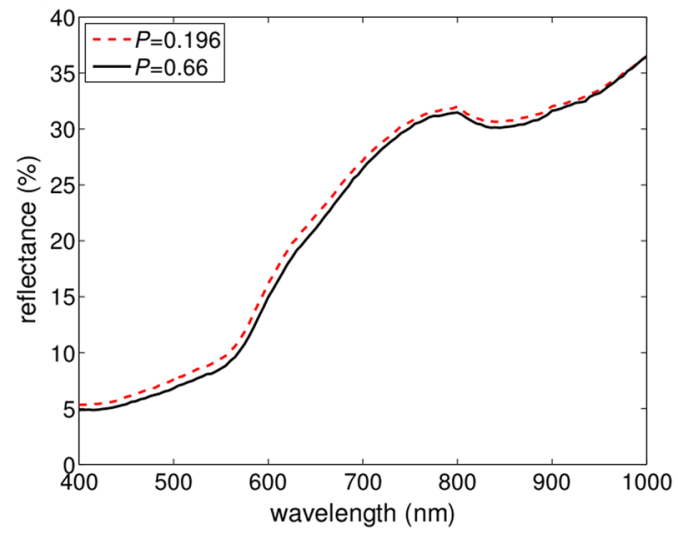

(b)

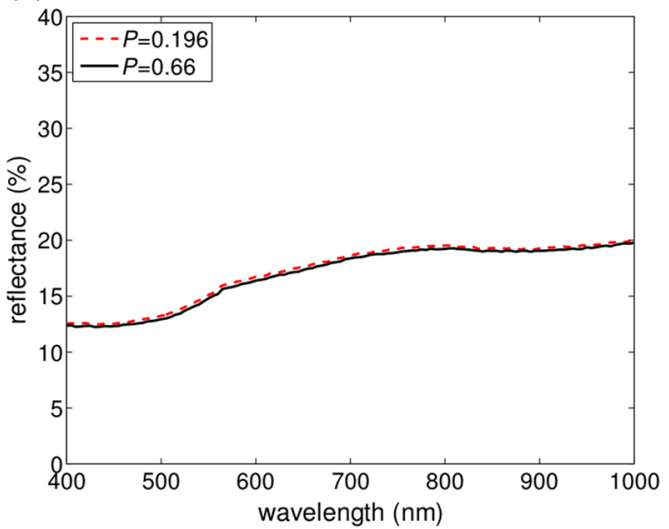

(d)

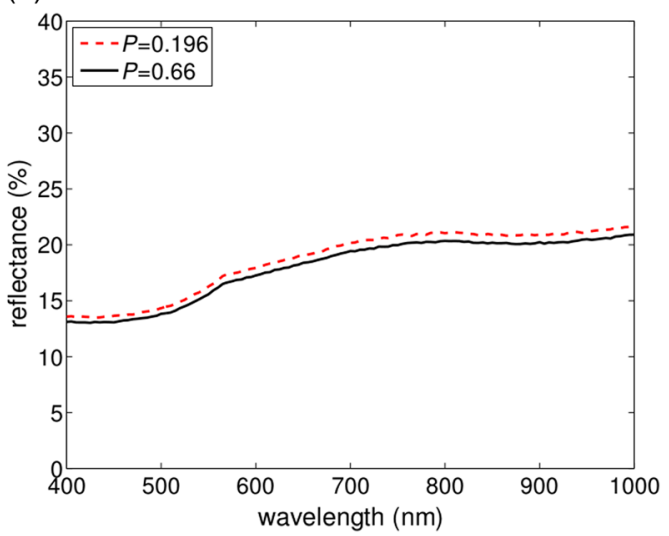

Fig. 4 Comparisons of modeled reflectance curves obtained for the Australian dune sample [(a) and (c)] and the Peruvian beach sample [(b) and (d)] considering variations in porosity $(P)$ and grain sphericity ( $\Psi$ ). (a) and (b) $\Psi=0.6$. (c) and (d) $\Psi=0.95$.

Table $5 \mathrm{MRD}_{\rho}$ values (given in percentages) computed for the modeled reflectance curves depicted in Fig. 4, which were obtained considering variations in porosity $(P=0.196$ and $P=0.66)$ and grain sphericity $(\Psi=0.6$ and $\Psi=0.95)$.

\begin{tabular}{|c|c|c|c|c|}
\hline \multirow{2}{*}{$\begin{array}{l}\text { Characterization } \\
\text { Parameter }\end{array}$} & \multicolumn{2}{|c|}{ Australian dune } & \multicolumn{2}{|c|}{ Peruvian beach } \\
\hline & Visible & Near-infrared & Visible & Near-infrared \\
\hline$\Psi=0.6$ & 4.95 & 5.37 & 1.98 & 1.25 \\
\hline$\Psi=0.95$ & 8.64 & 1.37 & 3.91 & 3.73 \\
\hline
\end{tabular}

$\Psi$ is set to 0.6 [Fig. 4(b)]. When $\Psi$ is set to 0.95 [Fig. 4(d)], however, one can observe a slightly more noticeable reflectance decrease along the entire spectral region of interest. These observations are corroborated by the corresponding $\mathrm{MRD}_{\rho}$ values provided in Table 5.

Simulations and experiments considering fine particulate media (diameters $<0.75 \mathrm{~mm}$ ) represented by assemblies of relatively uniform materials (e.g., granular cobalt glass or olivine basalt) reported in the literature ${ }^{20}$ indicated a decrease in reflectance in the visible domain following an increase in porosity. Although we have observed this trend in most of our light reflection experiments, we have also detected an increase in reflectance, notably outside the visible domain, in response to an increase in porosity when we considered upper and lower bounds for grain roundness [Fig. 3(c)] and sphericity [Fig. 4(a)], respectively. It is worth noting that those simulations and experiments reported in the literature ${ }^{20}$ neither took into account grain shape variations nor considered the complex mineralogical characteristics of the natural sands, 
particularly the presence of impurities (e.g., iron oxides characterized by distinct extinction coefficients) and their distribution patterns within these soils.

In all of our light reflection experiments (Figs. 2-4), a substantial variation in porosity from 0.196 to 0.66 resulted in relatively small reflectance changes (Tables 3-5). It is also worth mentioning that smaller variations in other sand characteristics, notably grain size and sphericity, result in significantly larger reflectance changes. ${ }^{22,24}$ Considering these aspects, the results of our in silico experiments demonstrate that, for natural sand deposits with typical mineralogical and morphological characteristics, the putative reflectance dependence on porosity in the spectral region of interest ${ }^{20}$ is markedly weaker than its dependence on these other sand characteristics.

\subsection{Light Transmission Experiments}

In our first set of light transmission experiments, we examined how the samples' transmittance is affected by the combined effects of variations in their porosity and degree of water saturation. The resulting plots depicted in Fig. 5 show the expected transmittance increase that follows an increase in the degree of water saturation. ${ }^{14,16,36}$ They also show an increase in transmittance following an increase in porosity. This trend can be explained by an increase in the sieve effect. ${ }^{42,43}$ This phenomenon occurs when light traversing a turbid medium does not encounter the main absorbers, with respect to the spectral region of interest, distributed within this medium. It reduces the probability of light absorption, notably with respect to the bands of absorption maxima of these absorbers. ${ }^{44-46}$ Consequently, more light is transmitted through the medium.

(a)

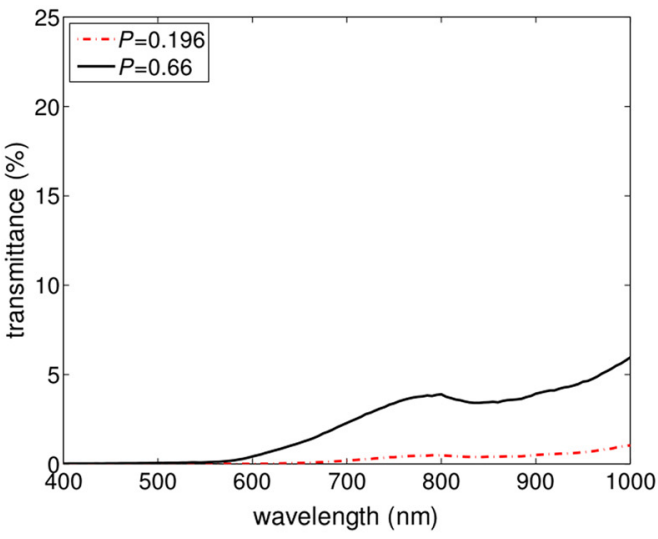

(c)

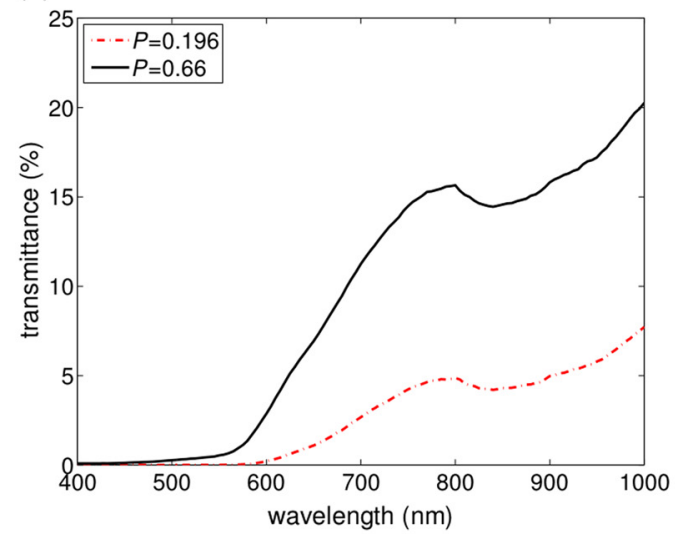

(b)

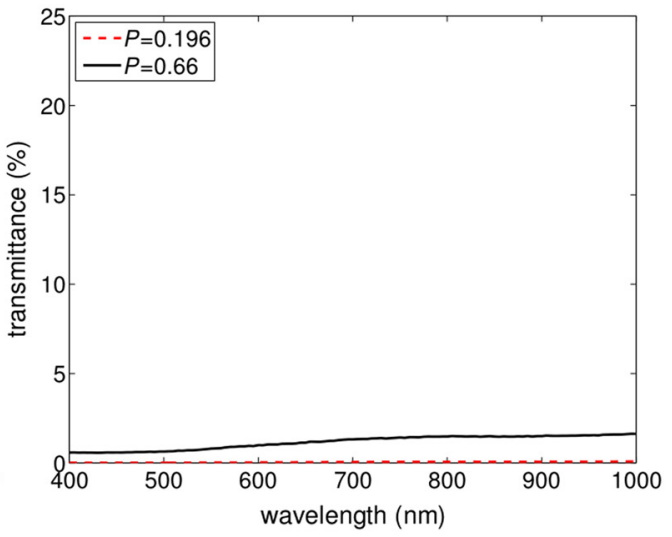

(d)

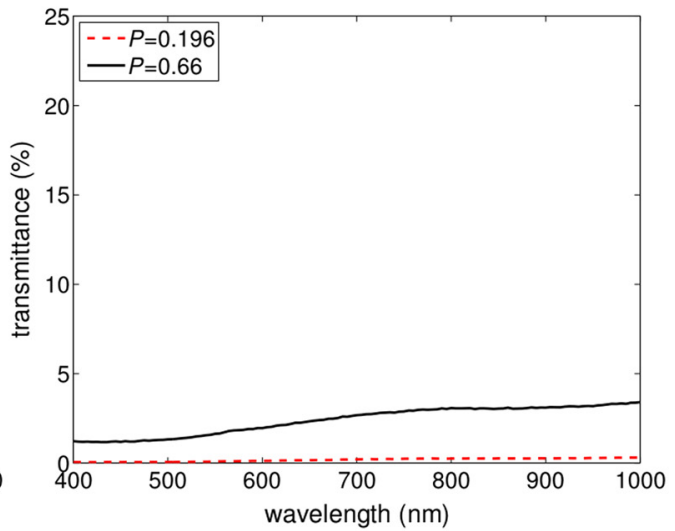

Fig. 5 Comparisons of modeled reflectance curves obtained for the Australian dune sample $[(\mathrm{a})$ and $(\mathrm{c})]$ and the Peruvian beach sample $[(\mathrm{b})$ and $(\mathrm{d})]$ considering variations in porosity $(P)$ and degree of water saturation (S). (a) and (b) $S=0$. (c) and (d) $S=1$. 
In the case of the selected samples, the main absorbers within the spectral region of interest are the iron oxides, namely hematite, goethite, and magnetite, whose bands of absorption maxima are located in the visible portion $\left(400\right.$ to $700 \mathrm{~nm}$ ) of this region. ${ }^{47-49}$ Hence, the impact on the transmittance curves should be larger in this spectral domain. This is corroborated by the corresponding $\mathrm{MRD}_{\tau}$ values provided in Table 6. Examining these values, we can also notice that the differences between the transmittance curves obtained considering $P$ equal to 0.196 and 0.66 are relatively smaller when $S$ is set to 1 . This may be explained by the fact that the presence of water in the pore space reduces the impact of the sieve effect by slightly increasing the probability of light absorption, ${ }^{43}$ particularly in the near-infrared portion (700 to $1000 \mathrm{~nm}$ ) of the spectral region of interest. We note that the extinction coefficient of water is significantly higher in the near-infrared than in the visible spectral domain. ${ }^{50-52}$

Table $6 \mathrm{MRD}_{\tau}$ values (given in percentages) computed for the modeled transmittance curves depicted in Fig. 5, which were obtained considering variations in porosity $(P=0.196$ and $P=0.66)$ and degree of water saturation $(S=0$ and $S=1)$.

\begin{tabular}{|c|c|c|c|c|}
\hline \multirow{2}{*}{$\begin{array}{l}\text { Characterization } \\
\text { Parameter }\end{array}$} & \multicolumn{2}{|c|}{ Australian dune } & \multicolumn{2}{|c|}{ Peruvian beach } \\
\hline & Visible & Near-infrared & Visible & Near-infrared \\
\hline$S=0$ & 97.98 & 87.47 & 97.16 & 95.58 \\
\hline$S=1$ & 93.51 & 69.14 & 94.57 & 91.61 \\
\hline
\end{tabular}

(a)

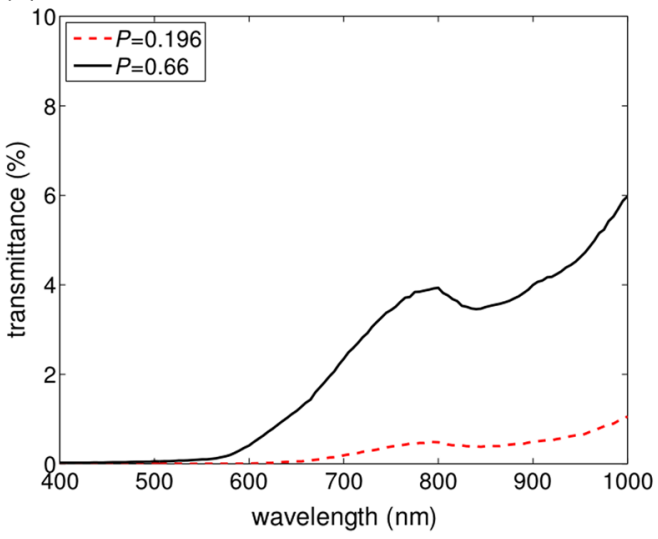

(c)

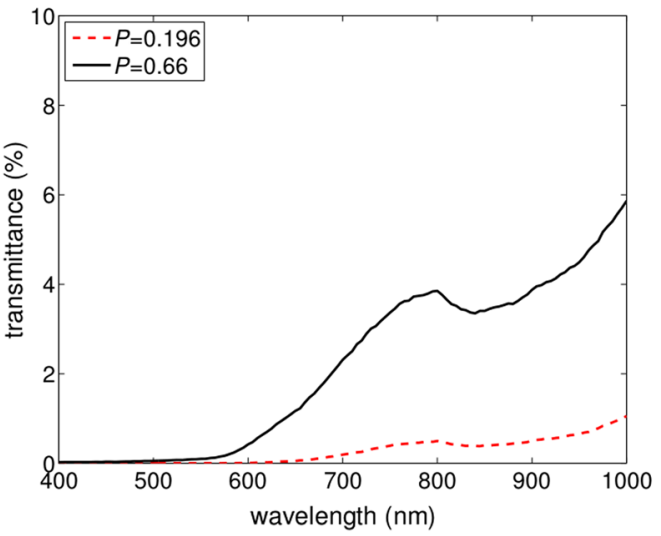

(b)

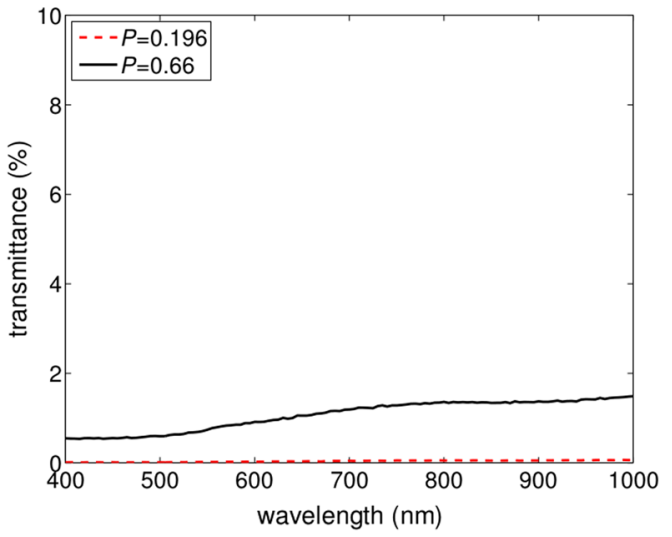

(d)

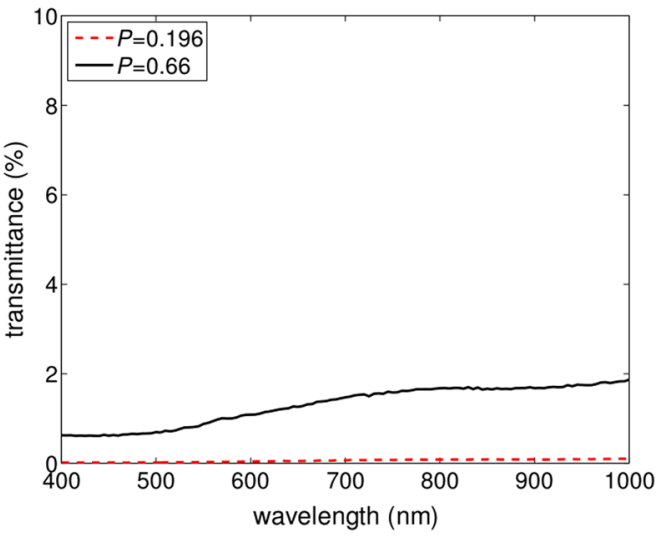

Fig. 6 Comparisons of modeled reflectance curves obtained for the Australian dune sample $[(\mathrm{a})$ and (c)] and the Peruvian beach sample [(b) and (d)] considering variations in porosity $(P)$ and grain roundness $(R)$. (a) and (b) $R=0.2$. (c) and (d) $R=0.7$. 
Table $7 \mathrm{MRD}_{\tau}$ values (given in percentages) computed for the modeled transmittance curves depicted in Fig. 6, which were obtained considering variations in porosity $(P=0.196$ and $P=0.66)$ and grain roundness $(R=0.2$ and $R=0.7)$.

\begin{tabular}{lccccc}
\hline \hline \multirow{2}{*}{$\begin{array}{l}\text { Characterization } \\
\text { Parameter }\end{array}$} & \multicolumn{2}{c}{ Australian dune } & & \multicolumn{2}{c}{ Peruvian beach } \\
\cline { 2 - 3 } \cline { 5 - 6 } & Visible & Near-infrared & & Visible & Near-infrared \\
\hline$R=0.2$ & 98.02 & 87.74 & & 97.45 & 96.11 \\
$R=0.7$ & 97.91 & 87.28 & & 96.71 & 94.95 \\
\hline \hline
\end{tabular}

In our second set of light transmission experiments, we examined how the samples' transmittance is affected by the combined effects of variations in their porosity and in the roundness of their constituent grains. The resulting plots depicted in Fig. 6 show again an increase in transmittance as $P$ is increased from 0.196 to 0.66 . These plots also show that an increase in $R$ from 0.2 to 0.7 had a minor impact on the transmittance curves. When we set $R$ to 0.7 , the relative differences between the transmittance curves obtained considering $P$ equal to 0.196 and 0.66 become slighter smaller than their counterparts calculated considering $R$ equal to 0.2 . This observation is corroborated by the corresponding $\mathrm{MRD}_{\tau}$ values provided in Table 7 .

In our third set of light transmission experiments, we examined how the samples' transmittance is affected by the combined effects of variations in their porosity and in the sphericity of their constituent grains. Similar to the previous sets of light transmission experiments, the resulting plots depicted in Fig. 7 show an increase in transmittance as $P$ is increased from 0.196 to

(a)

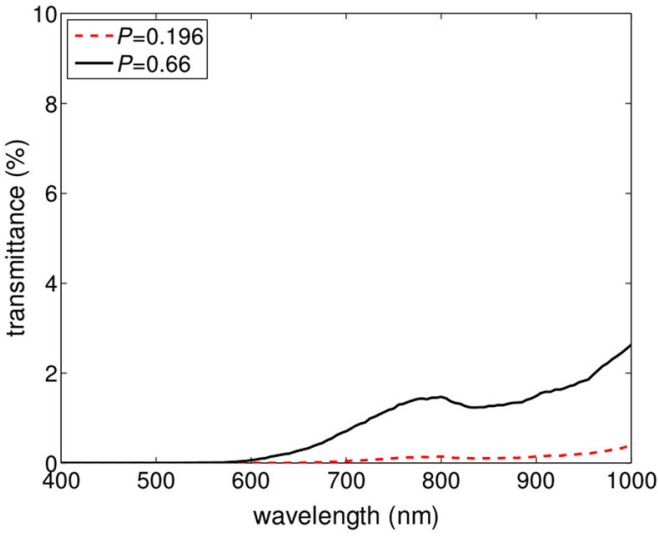

(c)

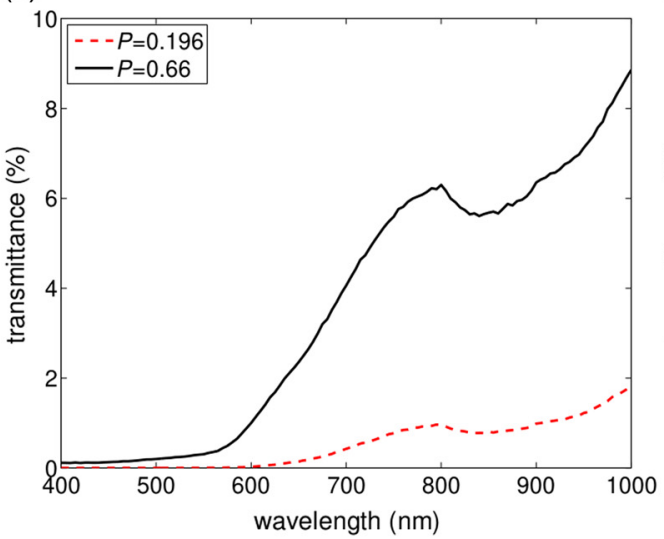

(b)

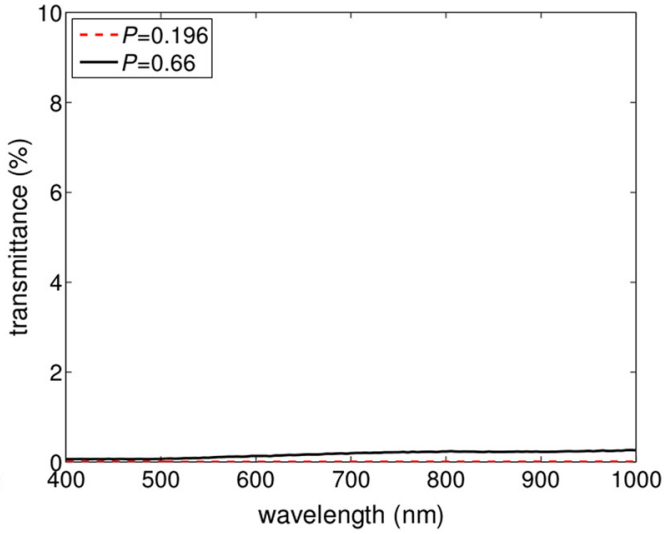

(d)

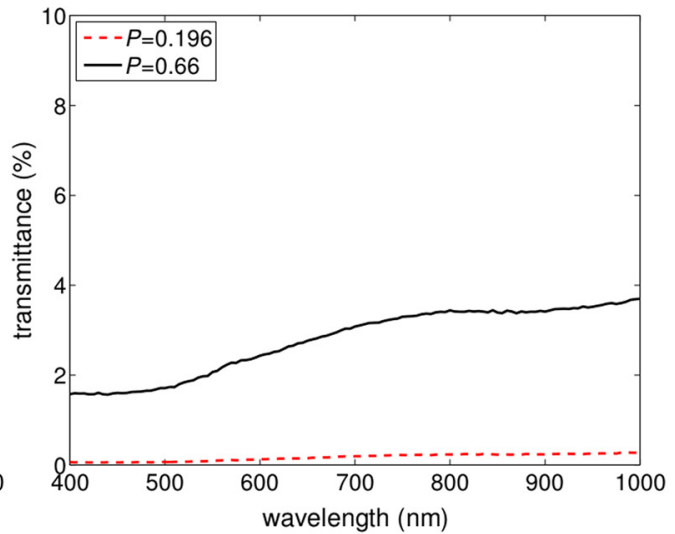

Fig. 7 Comparisons of modeled reflectance curves obtained for the Australian dune sample $[(\mathrm{a})$ and $(\mathrm{c})]$ and the Peruvian beach sample $[(\mathrm{b})$ and $(\mathrm{d})]$ considering variations in porosity $(P)$ and grain sphericity ( $\Psi$ ). (a) and (b) $\Psi=0.6$. (c) and (d) $\Psi=0.95$. 
Table $8 \mathrm{MRD}_{\tau}$ values (given in percentages) computed for the modeled transmittance curves depicted in Fig. 7 , which were obtained considering variations in porosity $(P=0.196$ and $P=0.66)$ and grain sphericity $(\Psi=0.6$ and $\Psi=0.95)$.

\begin{tabular}{|c|c|c|c|c|}
\hline \multirow{2}{*}{$\begin{array}{l}\text { Characterization } \\
\text { Parameter }\end{array}$} & \multicolumn{2}{|c|}{ Australian dune } & \multicolumn{2}{|c|}{ Peruvian beach } \\
\hline & Visible & Near-infrared & Visible & Near-infrared \\
\hline$\Psi=0.6$ & 98.21 & 90.53 & 98.07 & 97.33 \\
\hline$\Psi=0.95$ & 97.24 & 84.87 & 95.38 & 93.07 \\
\hline
\end{tabular}

0.66. These plots also show that an increase in $\Psi$ from 0.6 to 0.95 led to a noticeable increase in the transmittance curves. When we set $\Psi$ to 0.95 , the relative differences between the transmittance curves obtained considering $P$ equal to 0.196 and 0.66 become slighter smaller than their counterparts calculated considering $\Psi$ equal to 0.6 . This observation is corroborated by the corresponding $\mathrm{MRD}_{\tau}$ values provided in Table 8.

In all of our light transmission experiments (Figs. 5-7), a variation in porosity from 0.196 to 0.66 resulted in significant transmittance increases (Tables 5-7). These experiments have also shown that the compound impact of variations in porosity and degree of water saturation (Fig. 5) on transmittance curves can be noticeably larger than the impact of compound variations in porosity and grain roundness (Fig. 6). In addition, these experiments have indicated that the combined effects of variations in porosity and grain sphericity can lead to substantial transmittance changes (Fig. 7). We note that these trends were observed for both selected natural sand samples despite their mineralogical and morphological differences.

\subsection{Practical Issues}

It is worth highlighting that the realization of experiments free of biases associated with the physical handling of sand samples was among the main reasons for employing an in silico experimental approach in our investigation. We remark that sample alterations, such as grain breakage and grain-to-grain rearrangements, caused by the handling of these particulate materials can significantly affect their porosity. Moreover, these alterations can be detrimental to the accuracy of measured radiometric quantities, notably transmittance, directly associated with light penetration in the samples. Hence, they can also hinder the contributions of agricultural and ecological investigations that rely on sound estimations of the light penetration profiles of target sandtextured soils.

As with any investigation employing physically-based computer simulations, the outcomes of our light reflection and transmission experiments are still subject to practical confirmation. It is worth stressing, however, that we employed measured data reported in the literature, not only to characterize the selected natural sand samples, but also to guide the computation of their spectral responses. Moreover, we performed our in silico experiments using a first-principles light transport model, whose predictive capabilities have been qualitatively and quantitatively demonstrated in previous works. ${ }^{22,24,53-55}$ Hence, we are confident that our findings will be confirmed by actual spectrophotometric experiments as in situ measurement technologies capable of avoiding alterations in the samples' interstitial structure become readily available.

\section{Conclusion and Future Work}

Our findings suggest that advances in the remote estimation of sand deposits' porosity will require sensors with a high degree of sensitivity. Moreover, we note that previous works ${ }^{7,17}$ have indicated that the permeability of sand deposits may be correlated with grain size and sphericity in addition to porosity. Hence, new studies in this area, particularly those supported by remote sensing technologies, should take into account the potential masking effects that reflectance changes due to alterations in these grain characteristics may have on reflectance changes elicited by porosity variations. 
We remark that there is an extensive number of agricultural, ecological, and geophysical applications that are focused on the penetration of light in natural sands. These applications range from the germination of light-sensitive seeds ${ }^{14,15}$ and the photochemical transformation of toxic compounds, ${ }^{16}$ to the optical dating of sand deposits ${ }^{10,12}$ and the correct interpretation of remote signals originating from target materials covered by these soils. ${ }^{16,20}$ Our findings also indicate that initiatives in these areas will greatly benefit from the development of more effective tools for the estimation of porosity, especially given its impact on the amount of light that can penetrate these soils and reach relatively low depths of interest for these applications.

As future work, we plan to investigate the effects of porosity variations on the bidirectional surface scattering of natural sands. We also plan to extend our research to planetary regolith characterized by the occurrence of grains formed by distinct parent materials (e.g., basalt and silica-rich basaltic compositions).

\section{Acknowledgments}

This work was supported by the Natural Sciences and Engineering Research Council of Canada (NSERC-Discovery Grant No. 238337).

\section{References}

1. J. Gerrard, Fundamentals of Soils, Routledge, New York (2000).

2. N. Brady, The Nature and Properties of Soils, 8th ed., Macmillan Publishing Co., New York, (1974).

3. M. Shirazi, L. Boersma, and J. Hart, "A unifying quantitative analysis of soil texture: improvement of precision and extension of scale," Soil Sci. Soc. Am. J. 52(1), 181-190 (1988).

4. Soil Science Division Staff, "Soil survey manual," Technical Report, USDA Handbook 18, Soil Conservation Service, United States Department of Agriculture (1993).

5. R. Cooke and A. Warren, Geomorphology in Deserts, University of California Press, Los Angeles, California (1973).

6. N. Brady and R. Weil, Elements of the Nature and Properties of Soils, Prentice-Hall Inc., Upper Saddle River, New Jersey (2000).

7. J. Román-Sierra, J. Muñoz-Perez, and M. Navarro-Plus, "Beach nourishment effects on sand porosity variability," Coast. Eng. 83, 221-232 (2014).

8. W. Dickinson and J. Ward, "Low depositional porosity in eolian sands and sandstones, Namib desert," J. Sediment. Res. A64(2), 226-232 (1994).

9. P. Jackson, D. Smith, and P. Stanford, "Resistivity-porosity-particle shape relationships for marine sands," Geophysics 43, 1250-1268 (1978).

10. O. Lian, D. Huntley, and S. Wolfe, "Optical dating of eolian dune sand from Canadian prairies," Geogr. Phys. Quatern. 56(2-3), 191-202, 2002.

11. E. Chamberlaim, "A bright approach to geochronology," Phys. Today 71(9), 74-75 (2018).

12. J. Ollerhead, "Light transmittance through dry, sieved sand: some test results," Ancient TL 19(1), 13-17 (2001).

13. D. Koller, M. Sachs, and M. Negbi, "Germination-regulation mechanisms in some desert seeds VIII Artemisia monosperma," Plant Cell Physiol. 5, 85-100 (1964).

14. J. Woolley and E. Stoller, "Light penetration and light-induced seed germination in soil," Plant Physiol. 61, 597-600 (1978).

15. S. Benvenuti, "Soil light penetration and dormancy of jimsonweed (Datura stramonium) seeds," Weed Sci. 43, 389-393 (1995).

16. A. Ciani, K. Goss, and R. Schwarzenbach, "Light penetration in soil and particulate materials," Eur. J. Soil. Sci. 56, 561-574 (2005).

17. C. Curry et al., "Comparative study of sand porosity and a technique for determining porosity of undisturbed marine segment," Mar. Georesour. Geotechnol. 22, 231-252 (2004).

18. D. Tang et al., "Fine-scale volume heterogeneity measurements in sand," Soil Sci. Soc. Am. J. 27(3), 546-560 (2002). 
Baranoski, Kimmel, and Varsa: Assessing the impact of porosity variations on the reflectance...

19. M. Manchanda, M. Kudrat, and A. Tiwari, "Soil survey and mapping using remote sensing," Trop. Ecol. 43(1), 61-74 (2002).

20. B. Hapke, "Bidirectional reflectance spectroscopy 6. Effects of porosity," Icarus 195, 918-926 (2008).

21. G. Baranoski and B. Kimmel, "Can porosity affect the hyperspectral signature of sandy landscapes?" Proc. SPIE 10428, 104280S (2016).

22. B. Kimmel and G. Baranoski, "A novel approach for simulating light interaction with particulate materials: application to the modeling of sand spectral properties," Opt. Express 15(15), 9755-9777 (2007).

23. M. Vepraskas and D. Cassel, "Sphericity and roundness of sand in coastal plain soils and relationships with soil physical properties," Soil Sci. Soc. Am. J. 51(5), 1108-1112 (1987).

24. G. Baranoski et al., "Influence of sand-grain morphology and iron-oxide distribution patterns on the reflectance of sand-textured soils," IEEE J-STARS 7(9), 3755-3763 (2014).

25. J. Palmer, "The measurement of transmission, absorption, emission, and reflection," in Handbook of Optics (Volume II: Fundamentals, Techniques, \& Design), M. Bass et al., Eds., Optical Society of America, McGraw-Hill, Inc., New York, pp. 25:1-25:25, Chapter 25 (1995).

26. D. Catling and J. Moore, "The nature of coarse-grained crystalline hematite and its implications for the early environment of Mars," Icarus 165, 277-300 (2003).

27. A. Mottana, R. Crespi, and G. Liborio, Simon and Schuster's Guide to Rocks and Minerals, Simon and Schuster, Inc., New York (1978).

28. H. Wopfner and C. Twidale, "Australian desert dunes: wind rift or depositional origin?" Aust. J. Earth Sci. 48, 239-244 (2001).

29. H. Wopfner and C. Twidale, "Formation and age of desert dunes in Lake Eyre depocentres in central Australia," Geol. Rundsch. 77(3), 815-834 (1988).

30. F. El-Baz and D. Prestel, "Coatings on sand grains from Southwestern Egypt," in Desert Landforms of Southwest Egypt: A Basis for Comparison with Mars (NASA CR-3611), F. El-Baz and T. Maxwell, Eds., National Air and Space Museum Smithsonian Institution and National Aeronautics and Space Administration (NASA), Washington, DC, USA, pp. 175-188, Chapter 13 (1982).

31. R. Rossel et al., "Mapping iron oxides and the color of Australian soil using visible-nearinfrared reflectance spectra," J. Geophys. Res. 115, F04031 (2010).

32. D. Leu, "Visible and near-infrared reflectance of beach sands: a study on the spectral reflectance/grain size relationship," Remote Sens. Environ. 6(3), 169-182 (1977).

33. J. Rinker et al., "Remote sensing field guide—desert," Technical Report ETL-0588, U.S. Army Topographic Engineering Center, Fort Belvoir, VA, USA (1991).

34. G. Baranoski, J. Rokne, and G. Xu, "Virtual spectrophotometric measurements for biologically and physically-based rendering," Vis. Comput. 17(8), 506-518 (2001).

35. D. Bänninger and H. Flühler, "Modeling light scattering at soil surfaces," IEEE T. Geosci. Remote Sens. 42(7), 1462-1471 (2004).

36. M. Tester and C. Morris, "The penetration of light through soil," Plant Cell Environ. 10, 281-286 (1987).

37. Natural Phenomena Simulation Group (NPSG), "Run SPLITS online," David R. Cheriton School of Computer Science, University of Waterloo, Ontario, Canada (2012) http://www .npsg.uwaterloo.ca/models/splits.php.

38. G. Baranoski et al., "Rapid dissemination of light transport models on the web," IEEE Comput. Graph. 32(3), 10-15 (2012).

39. Natural Phenomena Simulation Group (NPSG), "Sand data," David R. Cheriton School of Computer Science, University of Waterloo, Ontario, Canada (2012).http://www.npsg .uwaterloo.ca/data/sand.php.

40. M. Baumgardner et al., "Reflectance properties of soils," Adv. Agron. 38, 1-44 (1986).

41. D. Lobell and G. Asner, "Moisture effects on soil," Soil Sci. Soc. Am. J. 66, 722-727 (2002).

42. L. Fukshansky, "Absorption statistics in turbid media," J. Quant. Spectrosc. Radiat. Transfer 38, 389-406 (1987).

43. D. Mandoli et al., "Some spectral properties of several soil types: implications for photomorphogenesis," Plant Cell Environ. 13, 287-294 (1990). 
44. L. Fukshansky, "On the theory of light absorption in non-homogeneous objects," J. Math. Biol. 6, 177-196 (1978).

45. P. Latimer, "A wave-optics effect which enhances light absorption by chlorophyll in vivo," Photochem. Photobiol. 40(2), 193-199 (1984).

46. T. Vogelmann, "Plant tissue optics," Annu. Rec. Plant Phys. 44, 231-251 (1993).

47. W. Eagan and T. Hilgeman, Optical Properties of Inhomogeneous Materials, Academic Press, New York (1979).

48. I. Sokolik and O. Toon, "Incorporation of mineralogical composition into models of the radiative properties of mineral aerosol from UV to IR wavelengths," J. Geophys. Res. 104(D8), 9423-9444 (1999).

49. A. Schlegel, S. Alvarado, and P. Wachter, "Optical properties of magnetite $\left(\mathrm{Fe}_{3} \mathrm{O}_{4}\right)$," J. Phys. C: Solid State Phys. 12, 1157-1164 (1979).

50. G. Hale and M. Querry, "Optical constants of water in the 200- nm to 200- $\mu \mathrm{m}$ wavelength region," Appl. Opt. 12(3), 555-563 (1973).

51. K. F. Palmer and D. Williams, "Optical properties of water in the near infrared," J. Opt. Soc. Am. 64(8), 1107-1110 (1974).

52. R. Pope and E. Fry, “Absorption spectrum (380-700 nm) of pure water. II. Integrating cavity measurements," Appl. Opt. 36(33), 8710-8723 (1997).

53. B. Kimmel and G. Baranoski, "A compact framework to efficiently represent the reflectance of sand samples," IEEE Trans. Geosci. Remote Sens. 47(11), 3625-3629 (2009).

54. B. Kimmel and G. Baranoski, "Simulating the appearance of sandy landscapes," Comput. Graph. 34(4), 441-448 (2010).

55. G. Baranoski et al., "Assessing the spectral sensitivity of martian terrains to iron oxide variations using the splits model," IEEE J-STARS 8(7), 3404-3413 (2015).

Gladimir V. G. Baranoski obtained his PhD in computer science at the University of Calgary (Canada) in 1998. Currently, he is a faculty member of the David R. Cheriton School of Computer Science at the University of Waterloo (Canada), where he has established the Natural Phenomena Simulation Group. His research interests include primarily the predictive simulation of light interactions with natural materials aimed at interdisciplinary applications. He is a member of SPIE.

Bradley W. Kimmel obtained his $\mathrm{PhD}$ in computer science at the University of Waterloo (Canada) in 2017. He received his MMath in computer science (2006) and BMath in pure mathematics and computer science (2002) from the University of Waterloo (Canada). His research interests include the simulation of light interaction with materials. He is currently working in the industry.

Petri Varsa is currently pursuing his $\mathrm{PhD}$ in computer science at the University of Waterloo (Canada). He received his master's of science degree from the University of Calgary in 2004 and his bachelor of mathematics degree from the University of Waterloo in 2001. His research interests include the simulation of natural phenomena and computer graphics. 